\title{
Analysis of stress influence on thermal diffusivity by photothermal infrared thermography
}

\author{
by H. Pron, J. F. Henry, S. Offermann, C. Bissieux and J. L. Beaudoin
}

Université de Reims, Unité de Thermique et Analyse Physique, Laboratoire d'Energétique et d'Optique, UFR Sciences, Moulin de la Housse, BP 1039, 51687 Reims Cedex 2, e-mail: jl.beaudoin@univ-reims.fr

\begin{abstract}
An infrared thermography equipment is used to measure the temperature rise at the surface of a steel bar, simultaneously submitted to the irradiation of a modulated laser beam and to a static uniaxial mechanical stress. The camera measures the radial temperature profiles across the laser beam, in order to point out the influence of stresses on the local thermal properties. Since this influence remains rather weak, a careful identification of the properties is to be undertaken.
\end{abstract}

\section{Introduction}

The present study is an attempt to point out mechanical stresses, applied or residual, by means of photothermal infrared thermography. The expected variations of thermal conductivity and specific heat as functions of stresses are estimated through their dependencies on temperature, together with the classical Hooke-Duhamel behaviour [1]. These temperature dependencies are available in the literature and few percent variations of thermal properties are to be expected within the elastic domain $[2,3]$.

The sample under investigation is submitted to the irradiation of a modulated laser beam, while it is loaded with a uniaxial tensile stress. The infrared camera measures the radial temperature profiles across the laser beam, which are more especially sensible to variations of thermal properties.

An axisymmetrical model of harmonic heat diffusion, using separation of variables, is used as the direct model. Then, a Gauss parameter estimation allows the identification of thermal parameters of the medium, with or without the influence of stress.

\section{A priori evaluation of the stress dependence of thermophysical properties}

Thermal conductivity and specific heat variations as functions of the stress state can be deduced from their dependencies on temperature, according to the thermoelastic behaviour law of the medium.

The derivation of Hooke-Duhamel's law leads to:

$$
\frac{\partial \sigma}{\partial T}=\frac{\partial \lambda}{\partial T} J_{1} \delta_{i j}+2 \frac{\partial \mu}{\partial T} \varepsilon_{i j}-\frac{\alpha E}{1-2 v} \delta_{i j}-\frac{\left(T-T_{0}\right)}{1-2 v} \delta_{i j}\left(E \frac{\partial \alpha}{\partial T}+\alpha \frac{\partial E}{\partial T}+\frac{2 \alpha E}{1-2 v} \frac{\partial v}{\partial T}\right)
$$

Neglecting the second order terms, and in the case of a static uniaxial loading $\left(\mathrm{J} 1=\sigma_{x x} / \lambda\right.$ and $\varepsilon_{\mathrm{ij}}=0$ ), we obtain, for stainless steel NC 22 D Nb (AFNOR) [1] :

$$
\frac{\partial \sigma}{\partial T}=3,6 \cdot 10^{-4} \sigma_{x x} \delta_{i j}-6,8 \cdot 10^{6} \delta_{i j}
$$

Within the elastic domain (e.g. for an applied stress of $100 \mathrm{Mpa}$ ) the first term remains far negligible and the stress variation versus temperature is

$$
\frac{\partial \sigma}{\partial T} \approx-6,8 \cdot 10^{6} \mathrm{~Pa} \cdot \mathrm{K}^{-1}
$$


On the other hand, the variation of the thermal diffusivity as a function of temperature is :

$$
\frac{\partial a}{\partial T}=\frac{k}{\rho C}\left[\frac{1}{k} \frac{\partial k}{\partial T}-\frac{1}{C} \frac{\partial C}{\partial T}-\frac{1}{\rho} \frac{\partial \rho}{\partial T}\right]=a\left[\frac{1}{k} \frac{\partial k}{\partial T}-\frac{1}{C} \frac{\partial C}{\partial T}-\frac{1}{\rho} \frac{\partial \rho}{\partial T}\right]
$$

Then, for stainless steel NC 22 D Nb (AFNOR) [1] ;

$$
\frac{1}{a} \frac{\partial a}{\partial T} \approx 10^{-3} K^{-1}
$$

The relative variation of thermal diffusivity as a function of stress is deduced as:

$$
\frac{1}{a} \frac{\partial a}{\partial \sigma}=\left(\frac{1}{a} \frac{\partial a}{\partial T}\right)\left(\frac{\partial T}{\partial \sigma}\right)
$$

Finally, for a stress variation about $100 \mathrm{MPa}$, a relative variation of thermal diffusivity is evaluated:

$$
\frac{\partial a}{a}=\frac{1}{a} \frac{\partial a}{\partial \sigma} \partial \sigma \approx-1,5 \cdot 10^{-2}
$$

Then, a few percent variation of the thermal properties is to be expected within the elastic domain $[2,3]$.

\section{Experimental setup}

A thin steel bar of $120^{*} 12^{*} 0.5 \mathrm{~mm}^{3}$ is simultaneously submitted to a static uniaxial tensile loading and to the irradiation of a mechanically modulated ion-argon laser. This laser is here used with an overall power of $1.5 \mathrm{~W}$ with Gaussian distribution, the $1 /$ e radius of which being $1.2 \mathrm{~mm}$, as monitored by a beam analyser. The only but essential preparation of the sample consists in the application of a $15 \mu \mathrm{m}$-thin black paint layer, in order to increase significantly its infrared emissivity.

Two mirrors, the first one spherical, the second one plane, are used to focus a real image of the steel bar surface in the object plane of the camera AGEMA $880 \mathrm{LW}$ (figure 1). The optical set-up brings a magnification of about 1 , in order to minimise the optical aberrations. The infrared camera is here used without optical complement, so observing a field of about $14 \mathrm{~mm}$, which is nearly the width of the bar.

Since the dimensions of the laser induced thermal spot depend on the excitation frequency via the thermal diffusion length $\mu$, the spatial sampling is well adapted for modulation frequencies of about $1 \mathrm{~Hz}$, for which $\mu$ is about $2 \mathrm{~mm}$ for steel. Under these conditions, the camera observes a scene including the laser spot and some diffusion lengths aside before reaching the sample boundary.

The thermal images are digitised with a standard 12 bits-acquisition system from National Instruments ${ }^{\mathrm{TM}}$, then treated by a home-made lock-in system developed with the. Labview ${ }^{\mathrm{TM}}$ software package, to obtain both amplitude and phase of the temperature. The phase origin is determined from a reference signal delivered by a photoelectric cell exposed to laser beam reflections on glass plates. Since the so-determined phase values are absolute ones, a numerical identification of thermophysical properties becomes conceivable.

\section{Identification procedure}

The identification of the thermophysical properties from the radial temperature profiles across the laser beam requires a direct model for the heat diffusion. An axisymmetrical model of harmonic heat diffusion, by the separation of variables, calculates the temperature inside a multi-layer sample irradiated by a Gaussian beam [4]. This thermal model is applied here to calculate the complex surface temperature profiles of a two-layer sample consisting 
in the metallic substrate and its black paint coating. In this configuration, the model shows namely that, outside the laser spot, the phase shift of the thermal signal increases at a rate of 1 radian per diffusion length of the substrate.

In order to solve the inverse problem, a two-parameter Gauss estimation [5] allows the identification of thermal conductivity and specific heat of the steel substrate. This inverse procedure is applied to temperature profiles on the same steel sample at different static stress levels. Considering that the density is known and independent of stress, the thermal diffusivity can then be calculated. The unknown parameters are estimated by comparison between the experimental temperature relative amplitudes and phase shifts and the values calculated by the thermal model. If the measurement noise presents a normal distribution, Student's test gives confidence intervals on the estimated parameters, in order to test whether or not their stress induced variations emerge from the noise. Moreover, the inverse method allows parameter sensitivity studies which can be used to optimise the experimental procedure and, when possible, to reduce the model.

\section{Results}

In order to validate both the experimental procedure and the identification routine, a first test was carried out on a metallic alloy reference sample. We determined a diffusivity value of $a=(3.7 \pm 0.1) 10^{-6} \mathrm{~m}^{2} \mathrm{~s}^{-1}$, which agrees quite fairly with the value measured by the classical rear-face flash method: $a=(3.6 \pm 0.1) 10^{-6} \mathrm{~m}^{2} \mathrm{~s}^{-1}$.

A crucial point of the diffusivity estimation procedure under $2 \mathrm{D}$ conditions is the evaluation of the actual magnification. Here, a value of 1.25 was experimentally determined by comparison between the bar- and the scene-width.

Then, the steel bar described above has been examined under a laser irradiation modulated at $0.9 \mathrm{~Hz}$, first without mechanical loading and then under a pure static traction. The strain rate of the loading was about $3 \cdot 10^{-3}$ and we controlled that the bar was not plastically deformed.

Figure 2 presents the phase shift profiles across the half laser spot, perpendicularly to the traction direction for these two stress states. A slight difference on the phase shift shapes can be observed. The difference on the phase value at the spot centre, about $20^{\circ}$ without loading and about $18^{\circ}$ under loading, is essentially due to the variation of the thermal conductivity, as attested by the sensitivity curves (figure 3 ). Indeed, the phase shift at the spot centre is more sensitive to the conductivity than to the specific heat.

Injecting these phase shift profiles in the parameter estimation procedure, a diffusivity value of $\mathrm{a}=(3.06 \pm 0.6) 10^{-6} \mathrm{~m}^{2} \mathrm{~s}^{-1}$ was estimated for the bar without loading and a value of $a=(2.85 \pm 0.6) 10^{-6} \mathrm{~m}^{2} \mathrm{~s}^{-1}$ for the bar under loading. The above uncertainties strictly arise from the identification routine and are given for $90 \%$ confidence intervals on both conductivity and specific heat. The overall uncertainties are necessarily larger, but the uncertainties on the other physical parameters should not be affected by the stress state variation.

According to these preliminary results, the application of strain seems here to modify the thermal diffusivity at a rate of about $5 \%$. However, this variation remains smaller than the uncertainties and needs further confirmations.

\section{Conclusion}

A first attempt has been undertaken in order to point out the influence of residual or applied stresses on the thermophysical properties of metallic samples. A slight variation has been actually observed but it remains smaller than the uncertainty interval.

More decisive results require an significant increase of the signal to noise ratio. Unfortunately, the magnitude of the stress that was applied here is not very far from the elastic limit. Consequently, the improvement effort has to be dedicated to the noise reduction. Firstly, the simpler way is to acquire much more profiles before the lock-in treatment. Moreover, the use of the amplitude profiles must yield more information, 
particularly in order to reduce the thermophysical parameter correlations. Finally, the spatial resolution will be enhanced by the implementation of a focal plane-array camera.

\section{REFERENCES}

[1] ROUBY (M.) and BLANCHARD (P.) - Propriétés physiques et mécaniques des aciers et alliages inoxydables, Les aciers inoxydables, Paris 1990, p. 111-160.

[2] MOUNTAIN (D. S.) and COOPER (G. P.) - TERSA-A new technique for assessing residual stress, Strain, 25, 1989, p. 15-19.

[3] DUNN (S. A.) and SPARROW (J. G.) - Stress dependence of specific heat : observations on the TERSA technique, Strain, 1990, p. 51-53.

[4] POTIER (F.) - Radiométrie photothermique appliquée à la caractérisation et au contrôle du traitement thermique des métaux, Université de Reims, 1989.

[5] BECK (J. V.) and ARNOLD (K. J.) - Parameter estimation in engineering and science, New York, 1977.

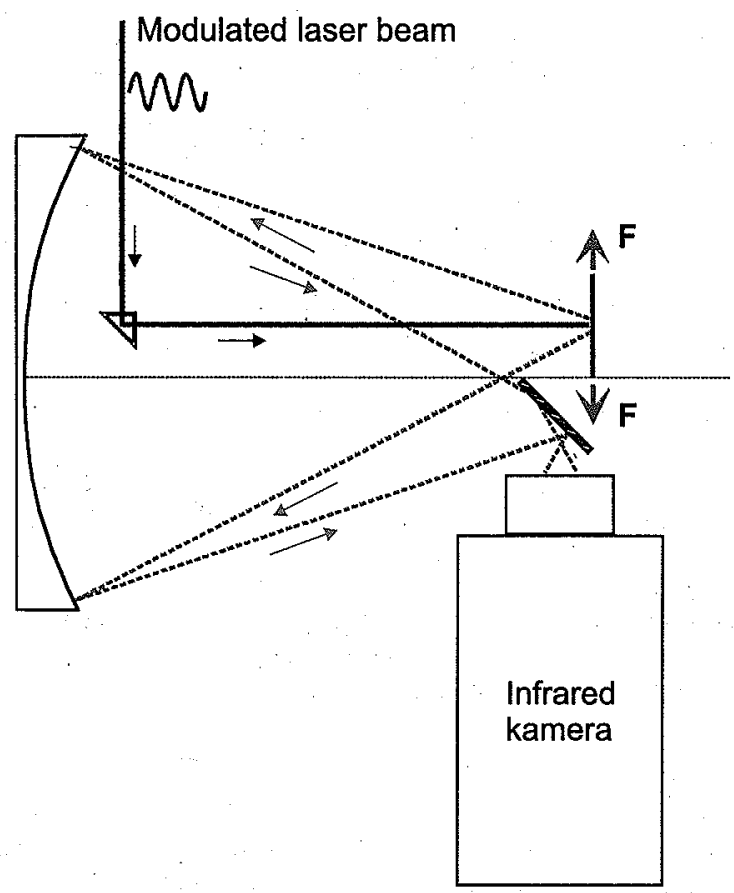

Fig. 1. Experimental setup 
http://dx.doi.org/10.21611/qirt.1998.020

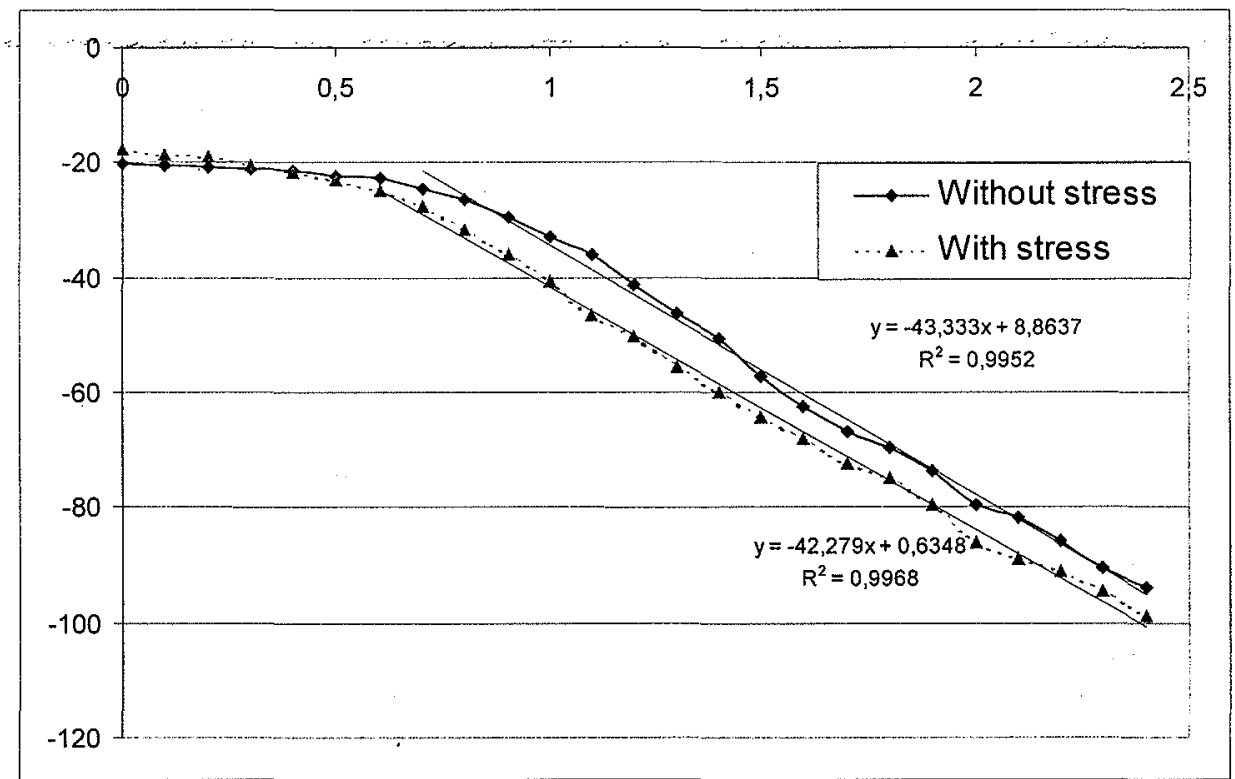

Fig. 2. Phase profiles

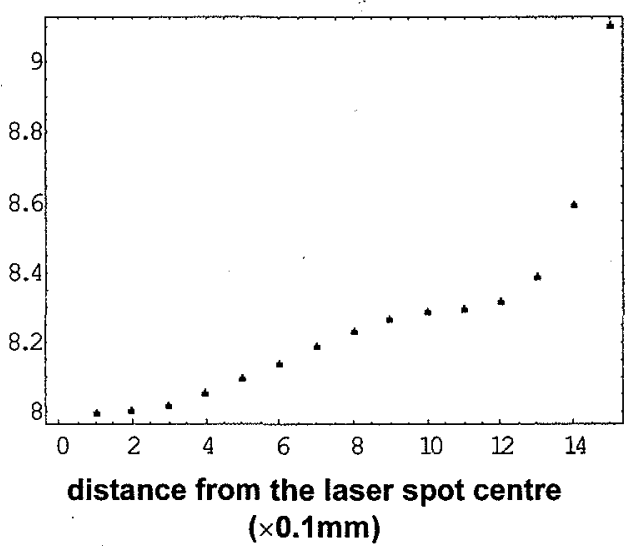

(a)

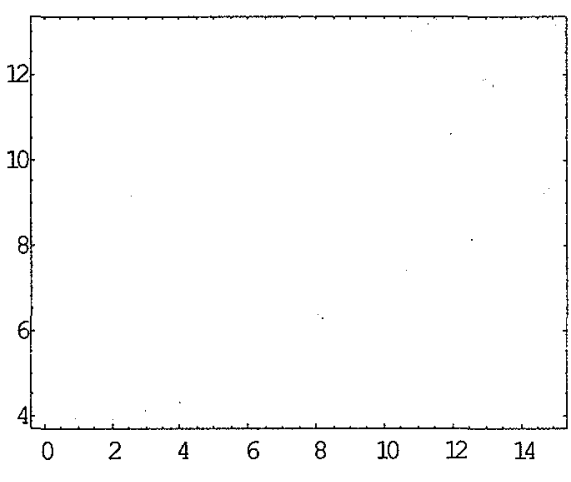

distance from the laser spot centre $(\times 0.1 \mathrm{~mm})$

(b)

Fig. 3. Sensitivity curves of the thermal conductivity (a) and of the specific heat (b) 